\title{
EFEITO DA ACETILAÇÃO DAS PARTÍCULAS DE MADEIRA DE Eucalyptus grandis E DA INCLUSÃO DE PARTÍCULAS DE POLIETILENO E DE EMBALAGENS CARTONADAS NAS PROPRIEDADES DE CHAPAS DE AGLOMERADO ${ }^{1}$
}

\author{
Fernando Vitor Oliveira², Benedito Rocha Vital ${ }^{3}$, José de Castro Silva ${ }^{3}$, Angelica de Cassia Oliveira
} Carneiro $^{3}$ e Alexandre Santos Pimenta ${ }^{3}$

\begin{abstract}
RESUMO - O objetivo deste trabalho foi avaliar o efeito da acetilação das partículas de madeira de Eucalyptus grandis e da inclusão de partículas de polietileno de baixa densidade (PEBD) e de alta densidade (PEAD) e de embalagens cartonadas nas propriedades de chapas de aglomerado. As partículas de madeira foram produzidas em laboratório a partir de toras que foram desdobradas em tábuas, convertidas em baguetas, depois em flocos e, finalmente, transformadas em partículas. Embalagens cartonadas e de polietino de alta e baixa densidades foram coletadas em depósitos de material reciclável e processadas em moinho de martelo. A acetilação foi realizada em autoclave com controle de pressão e temperatura. A acetilação promoveu significativa estabilização dimensional dos painéis, entretanto diminuiu algumas propriedades mecânicas. A adição de polietileno reduziu as propriedades mecânicas, em especial a flexão estática (MOR e MOE), porém, de modo geral, melhorou a estabilidade dimensional dos painéis, independentemente do tipo de partícula. No entanto, a adição de embalagens cartonadas reduziu a resistência mecânica e promoveu maior adsorção de vapor de água, inchamento em espessura e expansão linear, tanto nas chapas contendo partículas acetiladas quanto naquelas que não as continham.
\end{abstract}

Palavras-chave: Aglomerado, Acetilação, Polietileno e Embalagem cartonada.

\section{EFFECT OF PARTICLE ACETYLATION OF Eucalyptus grandis WOOD AND THE INCLUSION OF PARTICLES OF POLYETHYLENE AND CARDBOARD PACKAGING OF PROPERTIES IN CROWDED PLATES}

\begin{abstract}
ABSTRAC - The objective of this study was to evaluate the effect of eucalyptus grandis particles acetylation and the addition of polyethylene of low (pebd) and high density (pead) and paperboard particles in the properties particleboard. Wood particles were produced in laboratory from logs that were split into plaks, baguettes and then converted into flakes and finally transformed into particles. Paperboard and polyethylene collected in recyclable material stores and processed in a hammer mil. Acetylation was performed in autoclave with pressure and temperature control. Wood particles acetylation promoted a significant stabilization of the particleboards but decrease some mechanical properties. The addition of paperboard reduced all the mechanical properties and promoted a greater adsorption of water vapor, thickness swelling and linear expansion, for boards containing acetylated or non acetylated wood particles. The addition of polyethylene reduced the mechanical properties, specially the static bending (mor and moe), however resulted in more stable boards.
\end{abstract}

Keywords: Particleboard, Acetylation, Polyethylene and Paperboard.

\footnotetext{
${ }^{1}$ Recebido em 09.04.2008 e aceito para publicação em 02.03.2010.

${ }^{2}$ Programa de Pós-Graduaão em Ciência Florestal na Universidade Federal de Viçosa, MG, Brasil.

${ }^{3}$ Universidade Federal de Viçosa, MG, Brasil. E-mail: <bvital@ufv.br>, <cassiacarneiro@ufv.br>e<jcastro@ufv.br>.
} 


\section{INTRODUÇÃO}

Chapas de partículas de madeira aglomerada são produtos fabricados com pequenos fragmentos de material lignocelulósico, geralmente madeira, aglutinados com adesivos sintéticos ou outro aglomerante, sendo o conjunto termoprensado por tempo suficiente para que a lignina seja plasticisada e ocorra a cura do adesivo. Alem disso, é possível adicionar partículas de material lignocelulósico ou fibras vegetais e material termoplástico, produzindo-se os compósitos termoplásticos (YOUNGQUIST, 1995). Embora conhecidos desde o início do século $\mathrm{XX}$, os compósitos termoplásticos foram lançados no mercado em 1983 (CAULFIELD et al., 2005), quando começaram a ser fabricados no Japão e nos Estados Unidos, onde são denominados Wood Plastic Composite -WPC(YOUNGQUIST, 1995). Ainda segundo Youngquist (1995), eles têm a vantagem de serem renováveis, de baixo custo e não serem abrasivos. Além disso, as chapas de plástico-madeira podem ser fabricadas com resinas termoplásticas pós-consumo, contribuindo para reduzir a poluição ocasionada por essas resinas que, por não serem biodegradáveis, se tornam grande problema ambiental, podendo persistir no ambiente por centenas de anos (COELHO et al., 2008).

Em alguns produtos há a combinação de vários materiais, o que dificulta a sua reciclagem. É o caso das embalagens cartonadas (também conhecidas como "longa vida”, ou multicamadas ou, ainda, embalagens “tetra pak”, referência à empresa fabricante), compostas de polietileno de baixa densidade (PEBD), papelão e alumínio. Se viável, a incorporação de partículas de embalagem cartonada às partículas de madeira na fabricação de chapas de aglomerado seria outra forma capaz de reduzir a poluição do meio ambiente.

A fabricação de compósitos produzidos a partir de madeira e resíduos plásticos ou de embalagens cartonadas acompanha a tendência mundial de desenvolvimento sustentável, em que se buscam novos produtos a partir de matéria-prima alternativa, sendo um produto rotulado de "ecologicamente correto".

Além das vantagens do aglomerado tradicional, como a diminuição da anisotropia e a menor exigência de matéria-prima, podem se utilizar resíduos florestais; chapas produzidas pela combinação de madeira e resíduos plásticos agregam a outras vantagens. Entre estas se destaca o aumento da resistência à umidade e à deterioração por organismos biodeterioradores e o menor custo de manutenção (CORREA et al., 2003).
Uma dificuldade técnica a ser superada para o desenvolvimento da indústria de Wood Plastic Composites - WPC é a relação entre a madeira que apresenta caráter hidrofílico e o plástico de caráter hidrofóbico. Essa relação dificulta a adesão eficiente entre esses materiais, o que torna necessário o estudo de aditivos que possibilitem a obtenção de uma chapa de melhor qualidade (MACIEL, 2004ab; TEIXEIRA et al., 2002; CORREA et al., 2003). Além disso, mesmo que ocorra o encapsulamento das partículas de madeira, têm sido observadas a adsorção de vapor de água e a degradação biológica das partículas (MORRIS e COPPER, 1998; IBACH et al., 2004; SHIRP e WOLKOTT, 2005).

Uma possibilidade para melhorar a qualidade dos compósitos, em especial no que se refere à adesão entre as partículas, é a estabilização dimensional destas e, ou, o aumento da temperatura de prensagem que promovam a fusão do plástico, acarretando maior interação com a matriz madeireira.

Pode-se, também, melhorar a estabilidade dimensional da madeira e derivados, reduzindo os sítios de adsorção. Entre os vários processos químicos de tratamento da madeira com essa finalidade está a acetilação (ROWELL, 2007). Segundo Imamura et al. (1989), a adsorção de vapor de água pode ser reduzida, melhorando a estabilidade dimensional e a resistência à biodeterioração e acetilando-se as partículas de madeira. Contudo, segundo Rowell (2007), pode-se observar alguma redução nas propriedades das chapas de compósitos, porque a natureza hidrofóbica das partículas acetiladas pode diminuir a penetração dos adesivos.

Assim, o objetivo geral deste trabalho foi determinar as propriedades de chapas do tipo aglomerado fabricadas com partículas acetiladas e não acetiladas de Eucalyptus grandis, combinadas com partículas de polietileno de baixa densidade (PEBD) e alta densidade (PEAD) e de embalagens cartonadas, por termocompressão.

\section{MATERIAL E MÉTODOS}

Foram realizadas composições de partículas de madeira acetiladas e não acetiladas, polietileno de alta e baixa densidades e partículas de embalagem cartonadas.

A madeira de Eucalyptus grandis foi obtida em plantios comerciais pertencentes a empresas florestais localizadas nos Municípios de Ponte Alta, região do Vale do Rio Doce (latitude sul $19^{\circ} 42^{\prime}$ e longitude oeste $47^{\circ} 39^{\prime}$ ) e Três Marias, região de cerrado (latitude sul 
$18^{\circ} 12^{\prime}$ e longitude oeste $45^{\circ} 14^{\prime}$ ), no Estado de Minas Gerais. A massa específica básica média das madeiras foi igual a $560 \mathrm{~kg} / \mathrm{m}^{3}$

Os resíduos de embalagem cartonada e o polietileno de baixa densidade (PEBD) e de alta densidade (PEAD) foram obtidos, respectivamente, de um depósito de materiais recicláveis localizado no campus da UFV (ASBEN) e de uma recicladora de plásticos situada em Belo Horizonte, MG.

As toras de Eucalyptus grandis foram desdobradas, gerando tábuas com $1 \mathrm{~m}$ de comprimento e $2 \mathrm{~cm}$ de espessura, que pos-teriormente foram transformadas em baguetas com cerca de 6,00 cm de comprimento, que foram convertidas em flocos com 0,40 mm de espessura, 6,00 cm de comprimento e 2,00 cm de largura. Os flocos foram, novamente, processados em moinho de martelo, produzido-se partículas que foram classificadas, manualmente, em peneiras, mediante o uso de partículas que passaram pela peneira de 43,60 mm² e ficaram retidas na de $7,43 \mathrm{~mm}^{2}$. Em seguida, as partículas foram secas em estufa até atingir a umidade de aproximadamente 3\% e armazenadas em sacos plásticos até a sua utilização para produção das chapas.

As partículas de madeira a serem acetiladas foram secas em estufa aquecida a $103 \pm 3{ }^{\circ} \mathrm{C}$ até massa constante. Em seguida, foram pesadas e inseridas em autoclave, onde foram saturadas por $1 \mathrm{~h}$ com anidrido acético. O excesso de anidrido foi drenado e a autoclave aquecida a $120^{\circ} \mathrm{C}$, por $3 \mathrm{~h}$, conforme técnica descrita por Rowell et al. (1986). As partículas permaneceram na autoclave por mais $2 \mathrm{~h}$, com o equipamento desligado, e, posteriormente, foram deixadas ao ar livre, para evaporação do excesso de reagente. Foram, então, secas em estufa aquecida a $70 \pm 3^{\circ} \mathrm{C}$ até obter umidade base seca de aproximadamente 3\% e armazenadas em sacos plásticos até a produção dos painéis.

Os resíduos de embalagem cartonada e o polietileno de baixa densidade (PEBD) e de alta densidade (PEAD) foram obtidos, respectivamente, de um depósito de materiais recicláveis localizado no campus da UFV (ASBEN) e de uma recicladora de plásticos situada em Belo Horizonte, MG.

Os resíduos de polietileno de alta densidade (PEAD) e de baixa densidade (PEBD) foram fragmentados em moinho de martelo. Em seguida, as partículas foram classificadas, de forma semelhante às partículas de madeira, pela ação da gravidade e, posteriormente, armazenadas em sacos plásticos até a sua utilização para produção dos compósitos.

As embalagens cartonadas pós-consumo foram guilhotinadas e abertas para facilitar a lavagem. Em seguida, foram secas na temperatura ambiente e transformadas em partículas em moinho de martelo. Como as embalagens cartonadas contêm papel em sua constituição, as partículas foram secas em estufa aquecida a $70 \pm 3{ }^{\circ} \mathrm{C}$ até obter umidade base seca de aproximadamente $3 \%$.

A massa de partículas da mistura empregada na produção das chapas foi estimada de forma a permitir a produção de quatro chapas por tratamento, com dimensões de 10 x 400 x 400 mm e densidade final de aproximadamente $0,70 \mathrm{~g} \mathrm{~cm}^{-3}$. As partículas da mistura depois de pesadas foram colocadas em misturador tipo tambor rotatório, para aplicação da parafina e do adesivo, por meio de bico injetor, inserido no misturador e acionado por um compressor de ar. Empregaram-se 8\% de adesivo de fenolformaldeído (CASCOPHEN HL-2080 BORDENQuímica) e 0,5\% de uma emulsão de parafina sobre a massa seca de partículas.

Depois da aplicação do adesivo, a massa de partículas foi pesada e levada à seção formadora do colchão, em que as partículas foram manual-mente distribuídas sobre uma chapa de alumínio, para posterior prensagem. A prensagem ocorreu com temperatura de $170{ }^{\circ} \mathrm{C}$, em um ciclo de 8 min e taxa de compressão suficiente para obter chapas com $10 \mathrm{~mm}$ de espessura.

Após a prensagem as chapas foram entabicadas no Laboratório de Painéis e Energia do Departamento de Engenharia Florestal da UFV, em Viçosa, MG, até equilíbrio com o ambiente. Foram, então, esquadrejadas para 35,0 x 35,0 mm e suas superfícies, lixadas. Os corpos de prova foram retirados nas dimensões especificadas na Norma ASTM D-1037 (1991) e armazenados até equilíbrio em uma câmara climática a $20^{\circ} \mathrm{C}$ de temperatura e $65 \%$ de UR.

As propriedades foram comparadas com os valores mínimos exigidos pela Norma Comercial ANSI/ A - 208.1-1993.

O desenho experimental foi constituído por sete composições de partículas de madeira acetiladas e não acetiladas, polietileno de alta e baixa densidades e partículas de embalagem cartonadas com quatro

Revista Árvore, Viçosa-MG, v.34, n.5, p.937-946, 2010 
repetições, totalizando 56 painéis, conforme discriminado nas Tabelas 1 a 5 . Os resultados foram interpretados com o auxílio de análise de variância. Quando observado efeito significativo, as médias foram comparadas entre si, empregando-se o teste de Tukey a 5\% de probabilidade.

\section{RESULTADOS E DISCUSSÃO}

A acetilação média das partículas de eucalipto foi igual a 22\%, o que é compatível com o valor máximo observado por Rowell (1986) empregando a metodologia de acetilação utilizada neste trabalho. Não foram observadas diferenças significativas entre as densidades dos painéis, cujo valor médio foi de $0,71 \mathrm{~g} \mathrm{~cm}^{-3}$.

As umidades médias de equilíbrio das chapas depois de aclimatadas a $65 \%$ de umidade relativa e $20^{\circ} \mathrm{C}$ de temperatura, bem como a adsorção de umidade, quando as chapas passaram dessa condição de equilíbrio para 90\%, estão apresentadas na Tabela 1. Pode-se observar, nesse quadro, que as chapas contendo apenas partículas de madeira, seguidas pelas chapas contendo partículas de embalagens cartonadas, apresentaram umidades de equilíbrio higroscópico estatisticamente superiores às demais chapas, em cuja composição havia partículas de polietileno. Aumento na percentagem de partículas de polietileno ocasionou redução adicional na umidade de equilíbrio. As menores médias de umidade de equilíbrio das chapas contendo partículas de plástico se devem ao caráter hidrofóbico desse material e está de acordo com os resultados obtidos por outros pesquisadores (MACIEL, 2004ab; COSTA, 2003; MILAGRES, 2006). Também, a acetilação afetou significativamente a umidade de equilíbrio.

Todas as chapas contendo partículas de madeira acetiladas apresentaram valores médios de equilíbrio significativamente inferiores às médias daquelas fabricadas com partículas não acetiladas, observando-se redução na umidade de equilíbrio de até 48,1\%, em comparação com painéis contendo apenas partículas de madeira. O acréscimo de polietileno provocou redução adicional das médias de umidade de equilíbrio. As chapas contendo $60 \%$ de madeira e $40 \%$ de PEBD apresentaram, de modo geral, as menores médias de umidade de equilíbrio, enquanto o maior valor foi observado nas chapas com apenas partículas de madeira.

A inclusão de partículas acetiladas nos compósitos contendo embalagens cartonadas também ocasionou redução significativa no teor de umidade de equilíbrio higroscópico, em comparação com as chapas contendo partículas não acetiladas.

Tabela 1 - Valores médios de umidade de equilíbrio higroscópico e adsorção de vapor de água em função da acetilação e composição das partículas.

Table 1 - Mean values of equilibrium moisture content and water vapor adsorption as a function of acetylation and particle composition.

\begin{tabular}{|c|c|c|c|c|c|c|c|c|}
\hline \multirow{3}{*}{$\begin{array}{l}\text { Composição das } \\
\text { partículas }\end{array}$} & \multicolumn{2}{|c|}{ Partículas de Madeira } & \multirow{2}{*}{$\begin{array}{c}\text { Alteração }{ }^{1} \\
(\%)\end{array}$} & \multirow{2}{*}{$\begin{array}{l}\text { Média } \\
\text { Geral }\end{array}$} & \multicolumn{2}{|c|}{ Partículas de Madeira } & \multirow{2}{*}{$\begin{array}{c}\text { Alteração }{ }^{1 /} \\
(\%)\end{array}$} & \multirow{2}{*}{$\begin{array}{c}\text { Média } \\
\text { Geral }\end{array}$} \\
\hline & Não-acetiladas & Acetiladas & & & Não-acetiladas & Acetiladas & & \\
\hline & \multicolumn{4}{|c|}{ Umidade de equilíbrio higroscópico \% } & \multicolumn{4}{|c|}{ Adsorção de vapor de água \% } \\
\hline $1-100 \%$ Mad & $12,65 \mathrm{~A} \mathrm{a}$ & $6,56 \mathrm{~B}$ ab & 48,1 & 9,60 a & $2,28 \mathrm{~A} \mathrm{a}$ & $1,60 \mathrm{~B} \mathrm{a}$ & 29,8 & $1,94 \mathrm{ab}$ \\
\hline 2 - $80 \% \mathrm{Mad} / 20 \% \mathrm{PEBD}$ & $10,35 \mathrm{~A} \mathrm{c}$ & 5,39 B cd & 47,9 & $7,87 \mathrm{c}$ & $1,99 \mathrm{~A} \mathrm{~b}$ & $1,47 \mathrm{~B} \mathrm{a}$ & 26,1 & $1,73 \mathrm{~b}$ \\
\hline $3-60 \% \mathrm{Mad} / 40 \% \mathrm{PEBD}$ & $7,78 \mathrm{~A} \mathrm{e}$ & $4,41 \mathrm{~B} \mathrm{e}$ & 43,3 & $6,09 \mathrm{~d}$ & $1,77 \mathrm{~A} \mathrm{~b}$ & $1,57 \mathrm{~A} \mathrm{a}$ & 11,3 & $1,67 \mathrm{~b}$ \\
\hline $4-80 \% \mathrm{Mad} / 20 \% \mathrm{PEAD}$ & $9,57 \mathrm{~A} \mathrm{~d}$ & $5,84 \mathrm{~B} \mathrm{bc}$ & 39,0 & $7,70 \mathrm{c}$ & $2,03 \mathrm{~A} \mathrm{~b}$ & $1,67 \mathrm{~B} \mathrm{a}$ & 17,7 & $1,85 \mathrm{ab}$ \\
\hline $5-60 \% \mathrm{Mad} / 40 \% \mathrm{PEAD}$ & 7,88 A e & 5,05 B d & 35,9 & $6,47 \mathrm{~d}$ & $1,92 \mathrm{~A} \mathrm{~b}$ & $1,73 \mathrm{~A} \mathrm{a}$ & 9,9 & $1,82 \mathrm{ab}$ \\
\hline $6-80 \% \mathrm{Mad} / 20 \% \mathrm{EC}$ & 11,12 A bc & $6,24 \mathrm{~B} \mathrm{ab}$ & 43,9 & 8,68 b & $2,21 \mathrm{~A} \mathrm{a}$ & $1,83 \mathrm{~B} \mathrm{a}$ & 17,2 & 2,02 a \\
\hline $7-60 \% \mathrm{Mad} / 40 \% \mathrm{EC}$ & $10,69 \mathrm{~A} \mathrm{bc}$ & $6.55 \mathrm{~B} \mathrm{a}$ & 38.7 & $8.62 \mathrm{~b}$ & $2.27 \mathrm{~A} \mathrm{a}$ & $1.59 \mathrm{~B} \mathrm{a}$ & 30.0 & $1.97 \mathrm{ab}$ \\
\hline
\end{tabular}

Médias ao longo da mesma linha seguidas da mesma letra maiúscula e ao longo das colunas seguidas da mesma letra minúscula não apresentam diferença estatística significativa pelo teste de Tukey a 5\% de probabilidade. Em que Mad = madeira; PEBD = polietileno de baixa densidade; $\mathrm{PEAD}=$ polietileno de alta densidade; e EC = embalagem cartonada. ${ }^{1 /}$ (não acetiladas acetiladas)/não acetiladas.

Means along the same line followed by the same uppercase and along the columns followed by the same letter do not show statistically significant differences by Tukey test at 5\% probability. In that Mad = timber; LDPE = low density polyethylene, HDPE = high density polyethylene, and EC = carton. $1 /$ (non-acetylated-acetylated)/non-acetylated .

Revista Árvore, Viçosa-MG, v.34, n.5, p.937-946, 2010 
Analisando a média geral, observou-se que as chapas mais higroscópicas foram aquelas contendo 100\% de partículas de eucalipto ou partículas de eucalipto e embalagem cartonada.

As chapas que adsorveram maiores quantidades de vapor de água (Tabela 1) foram aquelas contendo $100 \%$ de partículas de madeira não acetiladas ou com partículas não acetiladas e embalagens cartonadas. A inclusão de polietileno ocasionou redução significativa na adsorção das chapas contendo partículas não acetiladas, porem não afetou a higroscopicidade das chapas fabricadas com partículas acetiladas.

A acetilação reduziu significativamente a adsorção de vapor de água, exceto nas chapas contendo 40\% de PEBD ou PEAD. Contudo, a média geral de adsorção dos painéis contendo partículas de PEBD foi inferior à adsorção das demais chapas. Isso indica que, de modo geral, como era esperado, a acetilação promoveu o bloqueio dos sítios de adsorção, responsáveis pela higroscopicidade da madeira, resultando em painéis menos higroscópicos.

Os valores médios de inchamento em espessura e expansão linear, ocasionados pelo aumento da umidade relativa de 65 para 90\%, são mostrados na Tabela 2 . A acetilação reduziu o inchamento em espessura em até 88,9\% e, a expansão linear, em até 50,0\%. Isso já era esperado, uma vez que a acetilação diminuiu a higroscopicidade das chapas. As chapas contendo partículas de madeira não acetiladas incharam menos quando contiveram em sua composição partículas de PEBD ou 40\% de PEAD. Contudo, a composição das partículas não afetou a expansão linear, exceto naquelas chapas fabricadas com partículas não acetiladas e 40\% de embalagem não cartonadas.

Considerando a média geral, observou-se que o inchamento da chapas contendo PEBD e 40\% de PEAD foi significativamente inferior ao das demais chapas. Esses resultados são coerentes com aqueles encontrados por outros pesquisadores, como Milagres (2006) e Cabral et al. (2006). As chapas menos estáveis foram aquelas fabricadas com 100\% de partículas de madeira ou 20 ou $40 \%$ de emba-lagens cartonadas.

A Tabela 3 contém os valores médios do módulo de ruptura e elasticidade em função do tipo e composição das partículas. Observa-se, nesse quadro, que os painéis contendo partículas não acetiladas e fabricados apenas com madeira, seguidos por aqueles contendo $20 \%$ de PEBD OU PEAD, tiveram a sua resistência à flexão estatisticamente superior à das demais composições. No entanto, as chapas contendo 40\% de PEBD ou $40 \%$ de embalagens cartonadas foram menos resistentes do que as demais.

De modo geral, à medida que se acrescentou polietileno ou embalagem cartonada, a resistência mecânica, bem como o modulo de elasticidade diminuiu, conforme indicado pela média geral. A acetilação reduziu

Tabela 2 - Valores médios de inchamento em espessura e expansão linear em função da acetilação e composição das partículas. Table 2 - Mean values of thickness swelling and linear expansion as a function of acetylation and particle composition.

\begin{tabular}{|c|c|c|c|c|c|c|c|c|}
\hline \multirow[t]{3}{*}{ Composição das partículas } & \multicolumn{2}{|c|}{ Partículas de Madeira } & \multirow{2}{*}{$\begin{array}{c}\text { Alteração }{ }^{\underline{1} /} \\
(\%)\end{array}$} & \multirow{2}{*}{$\begin{array}{l}\text { Média } \\
\text { Geral }\end{array}$} & \multicolumn{2}{|c|}{ Partículas de Madeiraa } & \multirow{2}{*}{$\begin{array}{c}\text { Alteração }{ }^{1} \\
(\%)\end{array}$} & \multirow{2}{*}{$\begin{array}{ll}1 & \text { Média } \\
& \text { Geral } \\
\end{array}$} \\
\hline & Não-acetiladas & Acetiladas & & & Não-acetiladas & $\overline{\text { Acetiladas }}$ & & \\
\hline & \multicolumn{4}{|c|}{ Inchamento em espessura \% } & \multicolumn{4}{|c|}{ Expansão linear \% } \\
\hline $1-100 \%$ Mad & $2,12 \mathrm{~A} \mathrm{a}$ & $0,30 \mathrm{~B} \mathrm{~b}$ & 85,8 & $1,21 \mathrm{~b}$ & 0,049 A bc & $0,040 \mathrm{~A} \mathrm{a}$ & 20,0 & $\overline{43 \mathrm{bc}}$ \\
\hline 2 - 80\% Mad/20\% PEBD & $1,55 \mathrm{~A} \mathrm{~b}$ & $0,25 \mathrm{~B} \mathrm{~b}$ & 83,9 & $0,90 \mathrm{c}$ & $0,055 \mathrm{~A} \mathrm{~b}$ & $0,030 \mathrm{~B} \mathrm{a}$ & 40,0 & 0,043 bc \\
\hline 3 - 60\% Mad/40\% PEBD & $1,17 \mathrm{~A} \mathrm{~b}$ & $0,24 \mathrm{~B} \mathrm{~b}$ & 79,5 & 0,70 с & 0,049 A bc & 0,039 A a & 20,0 & $0,044 \mathrm{bc}$ \\
\hline 4 - 80\% Mad/20\% PEAD & $2,16 \mathrm{~A} \mathrm{a}$ & $0,24 \mathrm{~B} \mathrm{~b}$ & 88,9 & $1,20 \mathrm{~b}$ & 0,051 A bc & 0,039 A a & 20,0 & $0,045 \mathrm{bc}$ \\
\hline 5 - 60\% Mad/40\% PEAD & $1,19 \mathrm{~A} \mathrm{~b}$ & $0,14 \mathrm{~B} \mathrm{~b}$ & 88,2 & $0,67 \mathrm{c}$ & $0,032 \mathrm{~A} \mathrm{c}$ & $0,026 \mathrm{~A} \mathrm{a}$ & 0,0 & 0,029 c \\
\hline $6-80 \% \mathrm{Mad} / 20 \% \mathrm{EC}$ & $2,12 \mathrm{~A} \mathrm{a}$ & $0,81 \mathrm{~B} \mathrm{a}$ & 61,8 & $1,46 \mathrm{ab}$ & 0,063 A b & $0,042 \mathrm{~B}$ a & 33,3 & $0,053 \mathrm{~b}$ \\
\hline $7-60 \% \mathrm{Mad} / 40 \% \mathrm{EC}$ & 2,09 A a & $0,95 \mathrm{~B} \mathrm{a}$ & 45,5 & 1,52 a & $0,100 \mathrm{~A} \mathrm{a}$ & $0,050 \mathrm{~B} \mathrm{a}$ & 50,0 & 0,075 a \\
\hline
\end{tabular}

Médias ao longo da mesma linha seguidas da mesma letra maiúscula e ao longo das colunas seguidas da mesma letra minúscula não apresentam diferença estatística significativa pelo teste de Tukey a 5\% de probabilidade. Em que Mad = madeira; PEBD = polietileno de baixa densidade; $\mathrm{PEAD}$ = polietileno de alta densidade; e EC = embalagem cartonada. ${ }^{\text {1/ }}$ (não acetiladas acetiladas)/não acetiladas.

Means along the same line followed by the same uppercase and along the columns followed by the same letter do not show statistically significant differences by Tukey test at 5\% probability. In that Mad = timber; LDPE = low density polyethylene, $H D P E=$ high density polyethylene, and EC = carton. $1 /$ (non-acetylated - acetylated) / non-acetylated.

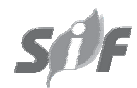

Revista Árvore, Viçosa-MG, v.34, n.5, p.937-946, 2010 
Tabela 3 - Valores médios de resistência à ruptura e modulo de elasticidade, respectivamente, em flexão estática em função da acetilação e composição das partículas.

Table 3 - Mean values of resistance and modulus of elasticity, respectively, in bending as a function of acetylation and particle composition.

\begin{tabular}{|c|c|c|c|c|c|c|c|c|}
\hline \multirow{3}{*}{$\begin{array}{l}\text { Composição das } \\
\text { partículas }\end{array}$} & \multicolumn{2}{|c|}{ Partícula de Madeira } & \multirow{2}{*}{$\begin{array}{c}\text { Alteração }{ }^{\underline{1}} \\
(\%)\end{array}$} & \multirow{2}{*}{$\begin{array}{l}\text { Média } \\
\text { Geral }\end{array}$} & \multicolumn{2}{|c|}{ Partícula de Madeira } & \multirow{2}{*}{$\begin{array}{c}{\text { Alteraçãa } \mathrm{O}^{\prime \prime}}^{(\%)} \\
\end{array}$} & \multirow{2}{*}{$\begin{array}{c}\text { 1/ } \text { Média } \\
\text { Geral }\end{array}$} \\
\hline & etiladas & Acetiladas & & & o-acetiladas & Acetiladas & & \\
\hline & \multicolumn{4}{|c|}{ Módulo de rupture - kgf/cm ${ }^{2}$} & \multicolumn{4}{|c|}{ Módulo de elasticidade - kgf/cm² } \\
\hline$\% 1$ & $A$ & $138,38 \mathrm{~B}$ ab & 305 & 168,7 & 164 & $21.487 \mathrm{~A} \mathrm{a}$ & 5.5 & 22.1 \\
\hline $\mathrm{o}_{\mathrm{c}}$ & b & & & & 19.1 & $\mathrm{~b}$ & & \\
\hline$-60 \% \mathrm{Mad} / 4$ & $\mathrm{Ae}$ & $\mathrm{d}$ & & & 13. & $3 \mathrm{~A} \mathrm{c}$ & $=$ & 13. \\
\hline$\%$ Mad/ & A & 14 & & & 20. & $3 \mathrm{~A} \mathrm{~b}$ & & 19 \\
\hline$\% \mathrm{Mad} /$ & 51 , & $11 \mathrm{~s}$ & & & 16.2 & B cd & & 15 \\
\hline$-\varepsilon$ & de & 126 & & & B cd & $\mathrm{A} \mathrm{b}$ & & 15. \\
\hline $7-60 \% \mathrm{Mad} / 40 \%$ EC & $8,12 \mathrm{~A} \mathrm{f}$ & A bc & -11 & $4 \mathrm{~d}$ & $10.737 \mathrm{~B}$ е & $13.325 \mathrm{~A} \mathrm{~cd}$ & $d-24,1$ & 11.981 \\
\hline
\end{tabular}

Médias ao longo da mesma linha seguidas da mesma letra maiúscula e ao longo das colunas seguidas da mesma letra minúscula não apresentam diferença estatística significativa pelo teste de Tukey a 5\% de probabilidade. Em que Mad = madeira; PEBD = polietileno de baixa densidade; $\mathrm{PEAD}$ = polietileno de alta densidade; e EC = embalagem cartonada. ${ }^{1 /}$ (não acetiladas acetiladas)/não acetiladas.

Means along the same line followed by the same uppercase and along the columns followed by the same letter do not show statistically significant differences by Tukey test at 5\% probability. In that Mad = timber; LDPE = low density polyethylene, $H D P E=$ high density polyethylene, and EC = carton. $1 /$ (non-acetylated - acetylated $) /$ non-acetylated .

de forma significativa em até $42 \%$ a resistência das chapas, exceto daquelas fabricadas com $40 \%$ de embalagem cartonada. O módulo de elasticidade foi reduzido em até $10,3 \%$, exceto naquelas fabricadas com embalagem cartonada que tiveram essa propriedade aumentada em até $24,1 \%$. Considerando apenas as chapas fabricadas com partículas acetiladas, observouse que aumento no teor de polietileno ou de partículas de embalagens cartonadas também reduziu o módulo de elasticidade. As chapas contendo 40\% de PEBD, ou PEAD ou, ainda, partículas de embalagens cartonadas apresentaram a menor resistência à flexão. Isso provavelmente tenha sido devido à menor adesão entre as partículas de madeira e de polietileno e embalagens cartonadas, o que causa deficiência na transmissão de esforços, assim como no teste de flexão estática. Alguns autores, como Cabral et al. (2006), correlacionaram a acetilação ou outros tratamentos que modificam os sítios de hidroxilas com a redução das propriedades de flexão estática. Isso ocorre porque, uma vez acetilados, esses sítios não podem mais interagir com o adesivo, acarretando adesão de menor resistência (OKINO et al., 1987).

Segundo a Norma ANSI/A - 1993, o valor mínimo do módulo de ruptura e elasticidade, para painéis com massa específica em torno de $0,70 \mathrm{~g} / \mathrm{cm}^{3}$, é igual a
$110 \mathrm{kgf} \mathrm{cm}^{-2} \mathrm{e} 17.250 \mathrm{kgf} \mathrm{cm}^{-2}$, respectivamente. Portanto, quando foram utilizados PEBD e partículas de madeira acetiladas, independentemente da proporção, a resistência à flexão foi inferior ao valor mínimo citado na norma. Chapas contendo $40 \%$ de embalagens cartonadas e partículas de madeira não acetiladas também não atingiram o valor mínimo da norma.

Considerando a média geral, observou-se que, além das chapas fabricadas com 100\% de partículas de madeira, os painéis com menor porcentual de plástico (20\% de PEBD e PEAD) apresentaram valores médios superiores ao mínimo exigido pela norma e foram significativamente superiores aos demais.

As resistência à tração perpendicular e arrancamento de parafuso são apresentadas na Tabela 4. Segundo a Norma ANSI/A - 93, a resistência mínima à tração perpendicular e arrancamento de parafuso é de $4,0 \mathrm{kgf} \mathrm{cm}{ }^{-2}$ e $90 \mathrm{kgf} / \mathrm{cm}^{2}$, respectivamente. A acetilação das partículas não afetou significativamente a resistência à tração perpendicular das chapas, exceto naquelas produzidas com $40 \%$ de PEBD ou contendo partículas de embalagem cartonada. Nesse caso, a acetilação aumentou essa propriedade em até $57,8 \%$. As chapas mais resistentes foram aquelas fabricadas apenas com partículas de madeira, acetiladas ou não acetiladas, ou, ainda, contento até $20 \%$ de PEBD ou PEAD.

Revista Árvore, Viçosa-MG, v.34, n.5, p.937-946, 2010 
Tabela 4 - Valores médios de resistência à tração perpendicular e arrancamento de parafuso, respectivamente, em função da acetilação e composição das partículas.

Table 4-Mean values of internal bond and screw withdrawal, respectively, as affected by acetylation and particle composition.

\begin{tabular}{|c|c|c|c|c|c|c|c|c|}
\hline \multirow{3}{*}{$\begin{array}{l}\text { Composição das } \\
\text { partículas }\end{array}$} & \multicolumn{2}{|c|}{ Partícula de Madeira } & \multirow{2}{*}{$\begin{array}{l}\text { Alteração }{ }^{1 / \prime} \\
(\%)\end{array}$} & \multirow{2}{*}{$\begin{array}{r}\text { Média } \\
\text { Geral } \\
\end{array}$} & \multicolumn{2}{|c|}{ Partícula de Madeira } & Alteração 1 & \multirow{2}{*}{$\begin{array}{r}\text { Média } \\
\text { Geral } \\
\end{array}$} \\
\hline & Não-acetilad & sAcetiladas & & & Não-acetiladas & Acetiladas & $(\%)$ & \\
\hline & \multicolumn{4}{|c|}{ Resistência à tração perpendicular } & \multicolumn{4}{|c|}{ Resistência ao arrancamento de parafuso } \\
\hline & \multicolumn{4}{|c|}{$\mathrm{kgf} / \mathrm{cm}^{2}$} & \multicolumn{4}{|c|}{ kgf } \\
\hline $1-100 \% \mathrm{Mad}$ & 8,79 A a & $8,76 \mathrm{~A} \mathrm{a}$ & 0,3 & 8,77 a & $136,38 \mathrm{~B}$ ab & $167,1 \mathrm{~A} \mathrm{a}$ & $-22,5$ & 151,74 \\
\hline 2 - $80 \% \mathrm{Mad} / 20 \%$ PEBD & 8,69 A a & 7,73 A ab & 11,0 & $8,21 \mathrm{ab}$ & 151,32 A a & 133,96 B b & 11,5 & 142,64 \\
\hline $3-60 \% \mathrm{Mad} / 40 \%$ PEBD & 4,69 B cd & 6,01 A bc & $-28,1$ & $5,35 \mathrm{c}$ & $130,30 \mathrm{~A} \mathrm{~b}$ & $96,0 \mathrm{~B} \mathrm{~d}$ & 26,3 & 113,2 \\
\hline $4-80 \% \mathrm{Mad} / 20 \%$ PEAD & $8,16 \mathrm{~A} \mathrm{a}$ & $8,09 \mathrm{~A} \mathrm{a}$ & 0,9 & $8,13 \mathrm{ab}$ & 135,75 B ab & 160,89 А а & $-18,5$ & 148,32 \\
\hline $5-60 \% \mathrm{Mad} / 40 \%$ PEAD & $7,89 \mathrm{~A} \mathrm{ab}$ & $7,49 \mathrm{~A}$ ab & 5,1 & $7,69 \mathrm{ab}$ & $149,12 \mathrm{~A} \mathrm{ab}$ & 140,53 A bc & 5,8 & 144,82 \\
\hline $6-80 \% \mathrm{Mad} / 20 \% \mathrm{EC}$ & 6,15 В bc & $7,72 \mathrm{~A} \mathrm{ab}$ & $-25,5$ & $6,94 \mathrm{~b}$ & 129,62 B b & $149,07 \mathrm{~A} \mathrm{ab}$ & $-15,0$ & 139,3 \\
\hline $7-60 \% \mathrm{Mad} / 40 \% \mathrm{EC}$ & $3,39 \mathrm{~B} \mathrm{~d}$ & $5,35 \mathrm{~A} \mathrm{c}$ & $-57,8$ & $4,38 \mathrm{c}$ & $102,62 \mathrm{~B} \mathrm{C}$ & $128,30 \mathrm{~A} \mathrm{c}$ & $-25,0$ & 115,46 \\
\hline
\end{tabular}

Médias ao longo da mesma linha seguidas da mesma letra maiúscula e ao longo das colunas seguidas da mesma letra minúscula não apresentam diferença estatística significativa pelo teste de Tukey a 5\% de probabilidade. Em que Mad = madeira; PEBD = polietileno de baixa densidade; $\mathrm{PEAD}=$ polietileno de alta densidade; $\mathrm{EC}$ = embalagem cartonada. ${ }^{1}$ (não acetiladas - acetiladas)/ não acetiladas.

Means along the same line followed by the same uppercase and along the columns followed by the same letter do not show statistically significant differences by Tukey test at $5 \%$ probability. In that Mad = timber; LDPE = low density polyethylene, $H D P E=$ High density polyethylene; EC = carton. $1 /$ (non-acetylated - acetylated) / non-acetylated .

Comparando a média geral das composições, observou-se que os painéis constituídos apenas de partículas de madeira apresentaram as maiores resistências à tração, entretanto sem apresentar diferença significativa em relação àquelas contendo $20 \%$ de plástico e 40\% de PEAD. Já as chapas fabricadas com partículas contendo $40 \%$ de PEBD e de embalagens cartonadas foram significativamente inferiores às demais.

Milagres (2006), trabalhando com madeira e polietileno de baixa e alta densidades, também concluiu que o acréscimo de plástico, em especial o PEBD, promove redução nos valores de tração. Isso pode ter sido ocasionado pela redução na taxa de compactação dos painéis à medida que se acrescentou plástico e pela maior dificuldade de adesão entre as partículas de plástico e madeira. Com a redução da taxa de compactação, espera-se diminuição dos valores de tração perpendicular, já que as partículas menos compactadas diminuíram os pontos de contato entre elas.

A resistência ao arrancamento de parafuso de todas as chapas foi superior ao valor mínimo estabelecido na Norma ANSI/A - 93. A acetilação das partículas de madeira ocasionou aumento significativo dessa resistência contendo apenas madeira, ou 20\% de PEAD ou, ainda, partículas de embalagem cartonada, aumentando essa propriedade em 22,5; 18,5; 15,0; e 25,0, respectivamente. No entanto, nas chapas com $40 \%$ de PEAD não houve diferença significativa, e naquelas fabricadas com PEBD 20 e $40 \%$ a acetilação diminuiu essa resistência em até 26,3\%.

Considerando a média geral, observou-se que as chapas contendo $40 \%$ de PEBD ou $40 \%$ de embalagem cartonada tiveram resistência significativamente inferior à das demais chapas. No entanto, conforme observado nas outras propriedades, o acréscimo de polietileno dificulta a adesão entre os materiais, dado o caráter hidrofóbico dos plásticos e hidrofílico da madeira. No caso de embalagens cartonadas, a geometria das partículas pode ter influenciado negativamente, uma vez que as partículas não apresentaram forma geométrica semelhante à de uma partícula de madeira, ou seja, não possuía o coeficiente de esbeltez adequado.

Os valores médios de Dureza Janka e compressão longitudinal estão apresentados na Tabela 5. Segundo a Norma ANSI/A - 1993, as chapas com densidade de 0,70 gr/ $\mathrm{cm}^{3}$ devem apresentar Dureza Janka mínima para essa propriedade de 222,5 kgf/ $\mathrm{cm}^{2}$, sendo todas as médias encontradas neste estudo superiores a esse valor.

Desdobrando as médias, não foram observadas diferenças significativas entre chapas fabricadas com partículas acetiladas e não acetiladas. Contudo, 
Tabela 5 - Valores médios de dureza Janka e compressão longitudinal, respectivamente, em função da acetilação e composição das partículas.

Table 5 - Mean values of hardness Jamka and longitudinal compression, respectively, as function of acetylation and particle composition.

\begin{tabular}{|c|c|c|c|c|c|c|c|c|}
\hline \multirow{3}{*}{$\begin{array}{l}\text { Composição das } \\
\text { partículas }\end{array}$} & \multicolumn{2}{|c|}{ Partícula de Madeira } & \multirow{2}{*}{$\begin{array}{c}\text { Alteração }{ }^{1 /} \\
(\%)\end{array}$} & \multirow{2}{*}{$\begin{array}{l}\text { Média } \\
\text { Geral }\end{array}$} & \multicolumn{2}{|c|}{ Partícula de Madeira } & \multirow{2}{*}{$\begin{array}{l}\text { Alteração }{ }^{\underline{1}} \\
(\%)\end{array}$} & \multirow{2}{*}{$\begin{array}{l}\text { Média } \\
\text { Geral }\end{array}$} \\
\hline & Não-acetiladas & Acetiladas & & & Não-acetiladas & Acetiladas & & \\
\hline & \multicolumn{4}{|c|}{ Dureza Janka - kgf/cm ${ }^{2}$} & \multicolumn{4}{|c|}{ Compressão longitudinal - kgf/cm² } \\
\hline $1-100 \%$ Mad & $502,75 \mathrm{~A} \mathrm{ab}$ & $516,25 \mathrm{~A}$ a & $-2,7$ & 509,5 a & $114,7 \mathrm{~A}$ a & $123,2 \mathrm{~A}$ a & $-7,4$ & 119,0 a \\
\hline $2-80 \% \mathrm{Mad} / 20 \% \mathrm{PEBD}$ & 496,0 A ab & 436,75 A bc & 11,9 & $466,38 \mathrm{~b}$ & $105,2 \mathrm{~A} \mathrm{ab}$ & $88,8 \mathrm{~A} \mathrm{~cd}$ & 15,6 & 97,0 bc \\
\hline $3-60 \% \mathrm{Mad} / 40 \% \mathrm{PEBD}$ & 401,75 A cd & 389,62 A c & 3,0 & 395,69 c & $79,2 \mathrm{~A} \mathrm{~cd}$ & 74,9 A d & 5,4 & $77,0 \mathrm{~d}$ \\
\hline $4-80 \% \mathrm{Mad} / 20 \% \mathrm{PEAD}$ & $526,25 \mathrm{~A}$ a & $495,17 \mathrm{~A} \mathrm{ab}$ & 5,9 & 510,71 a & $107,5 \mathrm{~A} \mathrm{ab}$ & $109,4 \mathrm{~A}$ ab & $-1,8$ & $108,5 \mathrm{ab}$ \\
\hline $5-60 \% \mathrm{Mad} / 40 \% \mathrm{PEAD}$ & $554,25 \mathrm{~A}$ a & $489,77 \mathrm{~A} \mathrm{ab}$ & 11,6 & 522,01 a & $96,6 \mathrm{~A}$ abc & 96,7 A bc & $-0,1$ & $96,6 \mathrm{bc}$ \\
\hline $6-80 \% \mathrm{Mad} / 20 \% \mathrm{EC}$ & 453,25 A bc & $476,77 \mathrm{~A} \mathrm{ab}$ & $-5,2$ & $465,01 \mathrm{~b}$ & $92,2 \mathrm{~A}$ bc & 92,6 A bcd & $-0,4$ & $92,4 \mathrm{c}$ \\
\hline $7-60 \% \mathrm{Mad} / 40 \% \mathrm{EC}$ & $371,0 \mathrm{~A} \mathrm{~d}$ & $469,83 \mathrm{~A} \mathrm{ab}$ & $-26,6$ & $420,42 \mathrm{c}$ & $69,8 \mathrm{~A} \mathrm{~d}$ & 73,7 A d & $-5,6$ & $71,8 \mathrm{~d}$ \\
\hline
\end{tabular}

Médias ao longo da mesma linha seguidas da mesma letra maiúscula e ao longo das colunas seguidas da mesma letra minúscula não apresentam diferença estatística significativa pelo teste de Tukey a 5\% de probabilidade. Em que Mad = madeira; PEBD = polietileno de baixa densidade; PEAD = polietileno de alta densidade; e EC = embalagem cartonada. ${ }^{1 /}$ (não acetiladas acetiladas)/não acetiladas.

Means along the same line followed by the same uppercase and along the columns followed by the same letter do not show statistically significant differences by Tukey test at 5\% probability. In that Mad = timber; LDPE = low density polyethylene, $H D P E=$ high density polyethylene, and EC = carton. $1 /$ (non-acetylated - acetylated) / non-acetylated.

verificaram diferenças significativas entre chapas produzidas com diferentes composições de partículas. Além disso, analisando a média geral, observou-se que a composição de partículas interferiu, de forma significativa, nos resultados. As chapas contendo apenas madeira ou madeira combinada com PEAD, nas proporções de 20 ou $40 \%$, foram mais resistentes do que as demais.

A Norma ANSI/A - 1993 não estipula valores mínimos para a resistência à compressão longitudinal. A acetilação não ocasionou alteração significativa nessa propriedade, que foi afetada apenas pela composição das partículas.

Os painéis compostos apenas por partículas de madeira apresentaram média igual à da composição com 20\% de PEAD e superior à das demais. As composições contendo madeira com 20\% de PEBD ou de embalagens cartonadas e madeira com $40 \%$ de PEAD não tiveram diferença entre as médias de compressão. Já as composições com maior proporção de PEBD e embalagens cartonadas mostraram médias inferiores às das demais composições.

\section{CONCLUSÕES}

A acetilação das partículas de madeira, ao bloquear os sítios de adsorção, permite a fabricação de chapas com maior estabilidade dimensional.
O acréscimo de polietileno também reduz os valores médios de umidade de equilíbrio, de adsorção e absorção de água, de inchamento em espessura e expansão linear. Entretanto, de modo geral, tanto a acetilação quanto a inclusão de partículas de polietileno podem reduzir algumas propriedades mecânicas das chapas.

O acréscimo de embalagens cartonadas resulta em chapas com elevada instabilidade dimensional e maior higroscopicidade e reduz a resistência mecânica.

É possível fabricar chapas do tipo aglomerado com partículas de madeira, acetiladas ou não acetiladas, combinadas com partículas de polietileno e obter propriedades satisfatórias, o que pode contribuir para incentivar a reciclagem de material pós-consumo.

\section{REFERÊNCIAS}

AMERICAN NATIONAL STANDARD - ANSI/A Mat-formed wood particleboard: Specification. ANSI/A 208.1 - 1993. National Particleboards Association, 1993. 9 p

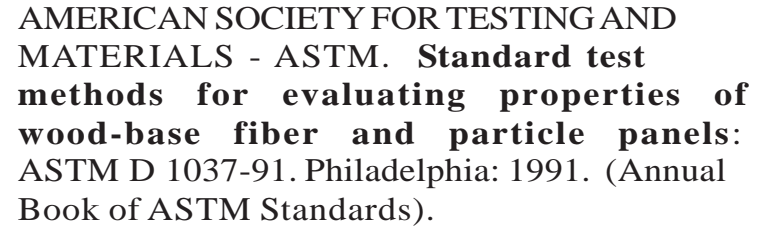


CABRAL, C.P.; VITAL,B.R.; DELLA LUCIA, R.M.; PIMENTA, A.S.; SOARES, C.P.; CARVALHO, A.M.M.L. Propriedades de chapas tipo OSB, fabricadas com partículas acetiladas de madeiras de Eucalyptus grandis, Eucalyptus urophilla, Eucalyptus cloeziana e Pinus elliottii. Revista Árvore, v.30, n.9, p.659-668. 2006.

CAULFIELD, D.; CLEAMONS, C.; JACOBSON, R.E.; ROWELL, R. M. Wood thermoplastic composites. In: ROWELL, R. M. Handbook of Wood Chemistry and Wood Composites. CRC Press, Boca Raton, 2005 p. 365- 378.

COELHO, N.S.; ALMEIDA, Y.B.; VINHAS, G.M. A biodegradabilidade da blenda de poli(âHidroxibutirato-co-Valerato)/amido anfótero na presença de microrganismos. Polímeros, v.18, n.3, 2008.

CORREA, C. A.; FONSECA, C. N. P.; NEVES, S.; RAZZINO, C. A.; HAGE, E. Compósitos termoplásticos com madeira. Ciência e Tecnologia, v. 13, n. 3, p. 154-165, 2003.

COSTA, D. M. L. Avaliação das propriedades físicas e mecânicas de compósitos plástico-madeira fabricados por termo-compressão, com partículas de eucalipto, polietileno tereftalato (PET) e poliestireno (PS). 2003.Dissertação (Mestrado em Ciência Florestal) - Universidade Federal de Viçosa, Viçosa, 2003.

IBACH, R.E.; CLEMONS, C.M.; STARK, N. Combined ultra-violet and water exposure as a preconditionioning method in laboratory fungal durability testing, In: Procedure of the $7^{\text {th }}$

International Conference on Woodfiber-Plastic composites. Forest Products Society, Madison, 2004.

IMAMURA,Y.SUBIYANTO,B.; ROWELL, R.M. NILSSON, T. Dimensional stability and biological resistance of particleboard from acetylated Albizzia wood particles. Wood Research, n.76, p. 49-58, 1989.

MACIEL, A.S.; VITAL, B.R.; DELLA LUCIA, R.M. PIMENTA, A.S. Painéis de partículas aglomeradas de Pinus elliottii Engelm., poliestireno (PS) e polietileno tereftatalato (PET). Revista Árvore, v.28, n.2, p.257-266, 2004.
MACIEL, A.S.; VITAL, B.R.; DELLA LUCIA, R.M. PIMENTA, A.S. Chapas de madeira aglomerada produzidas com partículas de Eucalyptus grandis W. Hill ex Maiden. Cerne, V.10, n.1, p.53-63, 2004.

MILAGRES, E. G. VITAL, B.R.; DELLA LUCIA, R. M. PIMENTA, A.S. Compósitos de partículas de madeira de Eucalyptus grandis, polipropileno e polietileno de alta e de baixa densidade. Revista Árvore, v.30, n.3, p.463-470, 2006.

MORRIS, P.I.; COPPER P. Recycled plastic/wood composite lumber attacked by fungy. Forest Products Journal, v.48, n.1, p.86-88. 1998.

OKINO, E.Y.A.; ANDAHUR, J.P.V.; SANTANA, M.A.E.; SOUZA, M.R. Resistência físicomecânica de chapas aglomeradas de bagaço de cana-de-açucar modificado quimicamente. Sciencia Forestalis, n.52; p.35-42, 1987.

ROWELL, R.M.; TILLMANN, A.M.; SIMONSON,R. A simplified procedure for the acetylation of hardboard and softwood flakes for flakeboard production. Journal of Wood Chemistry Technology, p.427-448, 1986 .

ROWELL, R. M.; Chemical modification o wood. In: FAKIROV, S; BHATTACHARYYA, D.;

Handbook of Engineering Biopolimers Hanser, Munich, 2007, p. 673- 691.

SHIRP ,A.; WOLCOTT, M.P. Influence of fungal decay and moisture absorption on mechanical properties of extruded wood-plastic composites. Wood and Fiber Science, v.37, n.4, p.643-652, 2005.

TEIXEIRA, D. E.; MOREIRA, J. M. M. A. P.; COSTA, A. F. Confecção de compostos de madeira-plástico utilizando resíduos de Eucalyptus grandis (Hill ex-Maiden) e polietileno de baixa densidade (PEBD). Floresta e Ambiente, v. 9, n. 1, p. 72-80, 2002.

YOUNGQUIST, J.A. Unlike partners? The marriage of woo and nonwood materials. Forest products Journal, v.45, n.10, 1995. 